\title{
Percepciones del emprendimiento con apoyo de herramientas tecnológicas: un enfoque descriptivo en tiempos de la COVID-19
}

\author{
Perceptions of entrepreneurship with the support of technological tools: a descriptive \\ approach in times of COVID-19 \\ DOI: $10.22458 /$ rna.v12i1.3380
}

PhD. Carlos Luis Chanto Espinoza ${ }^{1}$

MAP. Jorge Luis Loáiciga Gutiérrez²

${ }^{1}$ Universidad Nacional, Ingeniería en Sistemas de Información, Sede Regional Chorotega, Campus Liberia, Guanacaste, Costa Rica; carlos.chanto.espinoza@una.cr, https://orcid.org/0000-0002-3420-7259

${ }^{2}$ Universidad Nacional Costa Rica, Carrera de Administración, Sede Regional Chorotega, Campus Liberia, Guanacaste, Costa Rica; jorge.loaiciga.gutierrez@una.ac.cr, https://orcid.org/0000-0002-0421-3906

\section{RESUMEN}

Este artículo examina el emprendimiento frente a la pandemia del coronavirus. Se estableció una investigación, cuyo objetivo fue conocer las percepciones con respecto a emprender en tiempos de la COVID-19 con ayuda de herramientas tecnológicas para la orientación de los negocios en marcha y las propuestas de valor adaptables a la situación actual del mercado. La metodología se basa en el enfoque cuantitativo, de profundidad descriptiva, con estudio no experimental; se utiliza el cuestionario como herramienta de recolección de datos y la revisión bibliográfica, con una muestra constituida por 220 personas. Los resultados indican que, en medio de la pandemia, existe mucha incertidumbre al emprender $y$, ante la situación amenazante, surge la necesidad del apoyo en las herramientas tecnológicas, promovidas por el impulso de elementos como la motivación, el liderazgo, la búsqueda de capacitación y el asesoramiento financiero.

\begin{abstract}
This article examines entrepreneurship in the face of the coronavirus pandemic. Some research was established, whose objective was to discover the perceptions of undertaking in times of COVID-19, with the help of technological tools for the orientation of ongoing businesses and value proposals adaptable to the current market situation. The methodology is based on a quantitative approach, with a descriptive depth, with a non-experimental study, using the questionnaire as a data collection tool and a bibliographic review based on a sample of 220 people. The results indicate that in the midst of the pandemic there is a lot of uncertainty to undertake, and in the face of the threatening situation, there is a growing need for the support of technological tools promoted by the impulse of elements such as: motivation, leadership, the search for training and financial advice.
\end{abstract}

\section{RÉSUMÉ}

Cet article examine l'entreprenariat face à la pandémie du coronavirus. Une recherche a été établie dont l'objectif a été de connaître les perceptions en ce qui concerne le fait d'entreprendre en temps de COVID-19, avec l'aide d'outils technologiques pour l'orientation des entreprises en fonctionnement et les propositions de valeur adaptables à la situation actuelle du marché. La méthodologie est basée sur l'approche quantitative, de profondeur descriptive, avec une étude non expérimentale, en utilisant le questionnaire comme outil de collecte de données et la révision bibliographique, sur base d'un échantillon de 200 personnes. Les résultats indiquent qu'au milieu de la pandémie, il y a beaucoup d'incertitudes pour entreprendre, et face à la situation menaçante, surgit la nécessité du soutien des outils technologiques favorisés par l'impulsion d'éléments tels que : la motivation, la capacité de diriger, la recherche de la formation et le conseil financier.

\section{RESUMO}

Este artigo examina o empreendedorismo em face da pandemia do coronavírus. Foi estabelecida uma investigação com o objetivo de conhecer as percepções em relação a empreender nos tempos da COVID-19 com o auxílio de ferramentas tecnológicas para a orientação dos negócios em curso e propostas de valor adaptáveis à atual situação de mercado. A metodologia é baseada num enfoque quantitativo, de profundidade descritiva, com um estudo não experimental; $\mathrm{O}$ questionário é utilizado como instrumento de coleta de dados e revisão bibliográfica, com amostra composta por 220 pessoas. Os resultados indicam que, no meio à pandemia, há muita incerteza no momento de empreender e, diante da situação ameaçadora, surge a necessidade de apoio nas ferramentas tecnológicas, promovido pelo impulso de elementos como a motivação, a liderança, a procura de capacitação e assessoria financeira.
PALABRAS CLAVE:

EMPRENDIMIENTO,

CRISIS ECONÓMICA, TIC,

COVID-19, DESAFÍOS,

POLIITICAS DE ESTÍMULO

DEL EMPRENDIMIENTO.
KEYWORDS:

ENTREPRENEURSHIP

ECONOMIC CRISIS, ICT,

COVID-19, CHALLENGES,

POLICIES TO PROMOTE

ENTREPRENEURSHIP.
MOTS CLÉS:

ENTREPRENARIAT,

CRISE ÉCONOMIQUE,

TIC, COVID-19,

DÉFIS, POLITIQUES

D'ENCOURAGEMENT DE

L'ENTREPRENARIAT.
PALAVRAS-CHAVE:

EMPREENDEDORISMO,

CRISE ECONÔMICA,

TIC, COVID-19,

DESAFIOS, POLÍTICAS DE

ENCORAJAMENTO DO

EMPREENDEDORISMO 


\section{INTRODUCCIÓN}

La pandemia de la COVID-19 causó impactos inéditos en la totalidad del mundo, fundamentalmente en el contexto de la salud humana y en las acciones económicas. En este marco, el emprendimiento se creyó poco probable, tomando en cuenta elementos como el desplome en las economías del globo, cierre de compañías, pérdida de empleos, protestas mundiales, la amenaza invisible de un virus y pocos alicientes para avanzar.

La abrumadora realidad de la pandemia ha golpeado múltiples sectores de los negocios, sin respetar tipos de negocios o rotación de la industria; sus atronadores resultados económicos han causado el cierre de muchas compañías o, en el mejor de los casos, las han obligado a replantear su estructura para sobrevivir. Gran cantidad de empresas se vieron altamente afectadas a nivel económico y tuvieron la necesidad de despedir a centenas de colaboradores para no quebrar. No obstante, el sector más vulnerable ha sido el que conforman los emprendedores; primero, porque cuentan con negocios pequeños y, segundo, impulsan una nueva propuesta de valor en el mercado, la cual, de la misma forma, ha sido víctima de los trastornos económicos que se viven hoy. En este contexto, la utilización de las tecnologías de información y comunicación (TIC) como herramienta de apoyo se aceleró y dio paso para sostenerse, reducir costos y acrecentar la capacidad productiva de estos negocios.

El emprendimiento es entendido como la conducta del ser humano para adjudicarse riesgos y crear nuevos negocios. El emprendimiento constituye en la actualidad una de las atmósferas donde mayor incidencia tienen las TIC, al beneficiar bosquejos de globalización, disminuir distancias y alcanzar diversos mercados.

En relación con la huella de las TIC en el emprendimiento, varias investigaciones han justificado que la forma de emprender tiene diversos elementos, como lo es el tiempo en concordancia con el avance y evolución de la sociedad. Además, tanto las TIC como las transformaciones de los mercados benefician la creación de nuevos emprendimientos. De este modo, López y Rivera (2020) indican lo siguiente: "El emprendimiento en el mundo es un proceso sistémico importante que dinamiza la economía y genera fuentes de empleo” (p. 1).

Visto de esta forma, Chaves y Fonseca (2015) mencionan:

El emprendedurismo es un proceso de mucha importancia para el desarrollo económico de los países. A través de la creación de empresas, el progreso tecnológico y la innovación liderada por las personas emprendedoras es posible mejorar la creación de riqueza de una nación y coadyuvar con el bienestar de sus habitantes. Para ello se requiere de políticas públicas orientadas a mejorar las condiciones necesarias para que se desarrollen estas actividades emprendedoras. (p. 1)

Asimismo, Alean et al. (2017) señalan que “...el emprendimiento es el motor para el crecimiento económico de una nación, estos catalizan el cambio y la innovación al interior de los territorios, y se evidencia en el tejido empresarial con más y mejores empresas..." (p. 120).

En relación con este tema, Zamora-Boza (2017) argumenta:

El emprendimiento es un elemento clave del crecimiento económico. Pese a que los mecanismos exactos, la intensidad, la temporalidad y la direccionalidad de esta relación aún son desconocidas, es claro que la creación de empresas y la continuidad de estas en el tiempo aumentan la competencia, la generación de mejores empleos, la innovación y la diversificación productiva. (p. 24)

La COVID-19 ha impactado fuertemente la manera en que vivimos y ha transformado nuestra vida laboral y cotidiana. En esta perspectiva, Angelelli et al. (2020) afirman que "los efectos de la pandemia por la propagación de la COVID-19 están sacudiendo las estructuras socioeconómicas a nivel global. Los impactos se estiman en cifras astronómicas de pérdidas de vidas y empleos" (p. 1). Así mismo, las formas de adquirir productos y servicios, así como las necesidades de las personas, han cambiado. En tiempos de la COVID-19, los consumidores son más conscientes del gasto y las compañías tuvieron que adaptarse ágilmente a los cambios.

Cabe considerar, por otra parte, que la pandemia provocada por la COVID-19 ha creado oportunidades y ha apresurado procesos de transformación y permuta que llevaban aplazados algunos años, como el teletrabajo, la digitalización y la formación online. En este contexto, la denominada nueva normalidad está posicionándose en una crisis sanitaria, económica y social sin precedentes; los emprendimientos digitales, sanitarios y audiovisuales, entre 
otros, han crecido y han promovido proyectos de beneficio a raíz de nuevas dificultades y reformas en las pautas de consumo y ocio que el contexto de pandemia ha requerido.

El crear una empresa tiene su grado de dificultad y el coronavirus provocó la desaparición de innumerables compañías en todo el mundo. Por ende, el reinventarse apresuradamente, comprender las necesidades de los clientes y proyectar la vida dentro del contexto de la COVID-19 es sumamente significativo. Es aquí, en épocas de crisis, cuando el negocio informal es un fenómeno constante.

Las TIC inciden en forma significativa no solo en el desarrollo de las economías modernas, sino también en la afluencia de diversos escenarios que son parte del globo productivo. Sin duda alguna, nos referimos al escenario del emprendimiento empresarial, donde confluyen elementos financieros, condicionantes institucionales y las múltiples decisiones de los administradores para mantenerse en el mercado de modo competitivo. El emprendimiento es difícil y regularmente lo primero que se emprende son negocios de alimentos, ya sea en modo de comercio o por intermedio de un valor agregado.

En relación con la idea anterior, Félix y García (2020) indican:

Dichos negocios, por lo general, son de gestión familiar y no presentan una evolución muy satisfactoria de sus servicios y ratios económicos en los últimos años, razón por la cual deberán evolucionar y recalificarse por necesidad de supervivencia, especialmente en el uso de las tecnologías y los sistemas de comercialización digital. Igual es el caso del subsector de restauración, que deberá utilizar estrategias inteligentes para no perder su cuota de participación en el sector turístico y poder convertirse, como ocurre en muchos destinos, en uno de los más competitivos del mismo. (p. 97)

De igual forma, se debe mencionar el escenario de oportunidad, denominado globalización e innovación, el cual demanda gran creatividad. La pandemia ha puesto de manifiesto que los negocios deben ser más conectados, colaborativos, globales e innovadores. En relación con esto, Álvarez-Vásquez y Villafana-Del-Rosario (2020) establecen lo siguiente: "La explosión de la pandemia del nuevo coronavirus afectó -y sigue afectando-modelos de negocios con muchos años de sostenibilidad llevándolos casi a su extinción o adaptación para incurrir en nuevas formas de realizar sus labores" (p. 11).

\section{Objetivo general}

El objetivo de esta investigación se estableció en conocer las percepciones de emprender en tiempos de la COVID-19 con ayuda de herramientas tecnológicas para la orientación de los negocios en marcha y las propuestas de valor adaptables a la situación actual del mercado.

\section{FUNDAMENTACIÓN TEÓRICA}

En la actualidad, es muy común concentrarse en lo negativo. La propagación de la COVID-19 ha tenido un efecto catastrófico en la economía mundial, así como en la salud de los ciudadanos. El elemento novedoso de esta pandemia implica algunas incógnitas y es posible que presente un impacto perseverante durante los próximos años. A pesar del clímax actual, otras crisis históricas y pandemias sugieren un posible plan de recuperación para el mundo; pero, por el momento, la realidad es la abrumadora incertidumbre asociada a la COVID-19, la cual, además de afectar la salud mental y física de las personas, ha tenido una influencia demoledora en la economía familiar, ha provocado el cierre de muchos negocios o, en el mejor de los escenarios, los ha obligado a buscar la forma de reinventarse.

Tal como lo mencionan Jaramillo y Ñopo (2020):

La incertidumbre macro es también micro. En nuestra economía, de cada tres empleos, solo uno es formal; y de los otros dos, uno pertenece al ámbito del autoempleo. Los presupuestos familiares son altamente volátiles. Las restricciones a la libertad personal y económica que se requieren para enfrentar la emergencia sanitaria han significado, para muchos hogares, un corte abrupto de sus posibilidades de generar ingresos. (p. 12) 
En este panorama, surge la figura del emprendedor, quien, sin duda alguna, debe poseer la capacidad de negociar y para ello debe conectarse con los clientes y comunicar el precio de lo que está ofreciendo. Por ende, los sistemas de venta tradicionales, a consecuencia de la COVID-19, ya no marchan como antes y está claro que el emprendedor deberá examinar y modificar su forma de vender en la nueva normalidad. A esto se suma la necesidad de construir relaciones de confianza con redes y contactos que logren favorecer el emprendimiento. De la misma forma, el liderazgo es esencial, pues convendrá constituir y administrar equipos para lograr metas. En ese contexto, será muy significativo que todo emprendedor se comunique con claridad y fluidez, sumadas a la habilidad de escuchar a sus proveedores, clientes y entorno.

En relación con la problemática expuesta, Nicolás y Rubio (2020) afirman lo siguiente:

Los empresarios no siempre están preparados para afrontar periodos de crisis económicas, pero sin duda nadie se había preparado para los eventos de los últimos meses. Esto ha producido una paralización de gran parte de la actividad debido a los cierres temporales o definitivos de la actividad y los descensos de la demanda, generando a su vez una alta incertidumbre y una gran pérdida de capital humano. (párr. 3)

Dentro de este marco, ¿cuáles son las claves del emprendimiento en tiempos de pandemia? Sin duda alguna, el conocer a hondura el servicio o producto que se brinda es esencial y crea una ventaja en el tiempo al vender en forma remota, razón además para tener presencia en medios digitales a través de la red, desde su divulgación hasta el instante de cerrar la venta. Por ende, la interacción es fundamental para conservar un negocio en el entorno digital y establece como necesario que no solo se brinde el producto o servicio, sino que además tenga contenido y valor agregado, al gestionar con la ayuda de herramientas digitales como videollamadas y chats, entre otras.

Evidentemente, la COVID-19 obligó a los negocios a "cambiar la forma de llevar a cabo sus ventas. La ejecución de nuevas estrategias de comercio electrónico ayudará a estas compañías a sobrellevar la situación en la cual se encuentran y encontrarán mientras perdure la pandemia” (Mejías et al., 2020, p. 41).

En este sentido, muchas pymes emprendedoras han sido fuertemente afectadas por el impacto de la crisis en sus operaciones. En esta circunstancia, Vásquez-Lafebre et al. (2020) mencionan: "muchas de las medidas adoptadas por las organizaciones durante esta crisis, se convertirán en prácticas usuales, cambiarán los modelos de operación, la forma de trabajo será más ágil y colaborativa, se cambiarán los modelos de negocio” (p. 730).

Ahora bien, al revelar el impacto de las TIC sobre el emprendimiento, múltiples investigaciones han confirmado que la manera de emprender varía en el tiempo, lo cual insinúa la hipótesis de que las TIC y la desregulación de los mercados crean tendencias a lo largo del tiempo con respecto al nivel y la forma de creación de nuevas empresas. Sin embargo, esta conclusión es derivada de manera exógena por los trabajos señalados anteriormente, dado que analizan el impacto de las TIC sobre el emprendimiento a través de variables sociales para cada momento, como una manera de las tendencias tecnológicas de cada país.

Con respecto al papel de las TIC en el emprendimiento en tiempos de la COVID-19, Hoyos-Estrada y SastoqueGómez (2020) mencionan:

(...) esta crisis ha permitido acelerar un crecimiento en relación con el comercio en línea, el cual es aprovechado por aquellos negocios que cuentan con una fuerte presencia en Internet y han logrado una buena reputación online. El marketing digital se ha convertido en una herramienta efectiva para las grandes, medianas y pequeñas empresas, con el fin de obtener crecimiento en mercado globalizado. (p. 40)

Por otra parte, el impacto de las TIC no es lo único con lo que lidian los emprendedores más frágiles; estos se han enfrentado también a una serie de retos que en los meses de marzo a diciembre de 2020 se maximizaron a raíz de la pandemia. El desafío más importante recae en las situaciones económicas que muchas veces los han llevado a endeudarse; sin embargo, esta acción nada más solventa su situación en el corto plazo. Al respecto, Barrera et al. (2020) manifiestan: "en particular, los emprendedores y sus empresas pueden encontrar un entorno adverso al riesgo para nuevas opciones de inversiones, especialmente aquellas con modelos de negocio frágiles o con alta dependencia de algún tipo de insumo en particular" (p. 8). Asimismo, Vélez y Ortiz (2016) señalan que "algunos de los elementos 
principales que envuelven al emprendimiento e innovación son la creación, asumir riesgos, la motivación, las decisiones, el futuro, la oportunidad, y todos estos se relacionan al iniciar un proceso de emprendimiento con el mercado" (p. 348).

Linares (2020) expresa:

Siendo las pequeñas y medianas empresas, pymes, un tipo de organización que, si bien nutren buena parte del tejido empresarial de todos los países, independientemente del tamaño de sus economías, ellas adolecen de dirección y estructuras internas con capacidades suficientes para enfrentar los efectos económicos de una pandemia con las dimensiones del Covid 19, convirtiéndolas en entidades altamente vulnerables. De por sí, antes de la pandemia las pequeñas y medianas empresas eran uno de los eslabones más débiles de la globalización y un actor frustrado en su uso como instrumento de combate a la pobreza y desigualdad. (p. 4)

Por otra parte, el emprendimiento emerge como una estrategia para el desarrollo socioeconómico por su protagonismo en la generación de empleos, pese a todo el impacto provocado por la pandemia. De esta manera, Romero y Milone (2016) mencionan que "los emprendedores son los principales creadores de empleo facilitando la regeneración económica y social de los países" (p. 105). Sin embargo, al considerar lo anterior, hay que dejar claro que no se trata nada más de emprender, sino también de lo que implica para la economía de un lugar o de las familias, entre otros.

Desde esta perspectiva, González et al. (2018) afirman:

El emprendimiento no implica solo crear nuevas empresas. En el mundo actual, de gran complejidad tecnológica, con mercados en continua ebullición, tratados de libre comercio y la creciente toma de conciencia en todas las organizaciones sobre la necesidad urgente de innovar para sobrevivir, la presión para innovar y emprender se ha incrementado. (p. 8)

En este sentido se comprende que "el emprendimiento es un mecanismo que mediante la innovación incrementa la productividad, con la disponibilidad de los recursos humanos y tecnológicos adecuados" (Gonzaga et al., 2017, p. 323). De modo similar, Rodríguez (2016) establece que: "el emprendimiento es el proceso de realización de oportunidades con enfoque creativo, también es un factor importante para el desarrollo económico y como generador de cambio e innovación" (p. 422).

\section{METODOLOGÍA}

Para llevar a cabo el desarrollo del presente estudio, se utilizaron como referencia los métodos mixtos de investigación, pues incluye tanto particularidades del enfoque cuantitativo como del cualitativo, que "representan un conjunto de procesos sistemáticos, empíricos y críticos de investigación e implican la recolección y el análisis de datos cuantitativos y cualitativos, así como su integración y discusión conjunta, para realizar inferencias producto de toda la información recabada" (Hernández et al., 2014, p. 534).

Se empleó una profundidad de fase exploratoria-descriptiva, donde la fase de tipo exploratorio "es una investigación que se hace para obtener un primer elemento de una situación para luego profundizar y la fase descriptiva tiene el objetivo de describir los fenómenos" (Barrantes, 2015, p. 55). El alcance de esta investigación es longitudinal, debido a que los datos del estudio se recabaron en un tiempo determinado, además de ser de tipo no experimental. Las técnicas para la recolección de datos fueron la encuesta, la observación y la revisión bibliográfica.

La muestra total considerada fue de 220 personas, conformadas por 98 mujeres y 122 hombres de entre 20 y 39 años de edad, pertenecientes a la provincia de Guanacaste, principalmente de los cantones de Liberia, Carrillo y Upala, con estudios universitarios, secundarios y técnicos.

Para recopilar la información, se utilizó un cuestionario con preguntas cerradas y abiertas, diseñado en la plataforma tecnológica de Google Form y compartido por medio de las aplicaciones digitales de WhatsApp y Messenger de Facebook. Se utilizó estadística descriptiva con frecuencias tanto porcentuales como absolutas durante el análisis de resultados. Los cuestionarios fueron aplicados del 5 de diciembre de 2020 al 5 de enero del 2021. 


\section{RESULTADOS}

Tras identificar los efectos de la COVID-19 sobre la situación actual, uno de los resultados más relevantes es que cerca del 66,2 \% (145 personas en términos absolutos) se encuentran ligadas a una situación de tipo asalariado, es decir, aún no se desligan por completo para desarrollar su idea de negocio, con el argumento de considerar situaciones de inseguridad y miedo a la incertidumbre que se agravó con la pandemia.

Dentro de este orden de ideas, se consultó a las personas encuestadas cuáles elementos consideraban beneficiosos de recibir para el desarrollo de un emprendimiento con ayuda de herramientas tecnológicas, a lo cual el 32,3\% se inclinó por la asesoría con respecto a marca, planificación y costos; el 15,9 \% a la realimentación del proyecto por un mentor y experto; un 14,5\% a las charlas con expertos nacionales e internacionales y el $14,1 \%$ a recibir cursos online. Otros pocos mencionaron lecturas de libros, diseños para marketing, testimonios de emprendedores, talleres y financiamientos. En la figura 1 se ilustran los elementos sugeridos:

\section{Figura 1}

Percepción de elementos propuestos para emprender con herramientas tecnológicas

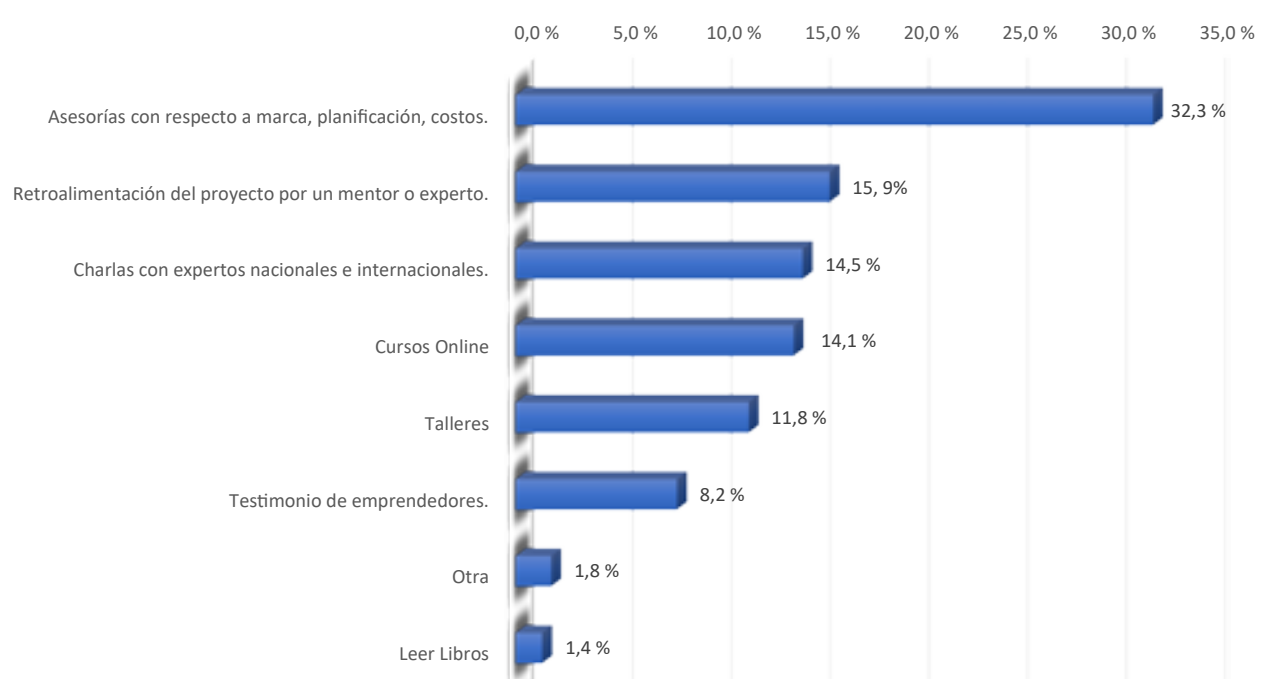

Fuente: elaboración propia.

Los sujetos encuestados consideran que en un mercado cada día más digitalizado se necesita desarrollar una cultura emprendedora de la mano con las herramientas tecnológicas; 192 encuestados consideran que les hace falta conocimiento en herramientas digitales gratuitas, dinero, asesoramiento financiero y conocimientos en administración de proyectos. Por otro lado, 28 personas encuestadas indicaron la necesidad de mejorar en su motivación y contar con bases bibliográficas de lectura sobre proyectos exitosos e información sobre emprendedurismo, la cual muchas veces es adquirida con base en la experiencia, con episodios de prueba y error. 
Cabe considerar, por otra parte, que, en las personas que completaron el cuestionario, los siguientes elementos: bases de datos bibliográficas de proyectos realizados, dinero, motivación, comunicación con expertos, asesoramiento financiero, apoyo o guía de un mentor y experiencia profesional previa a emprender fueron evaluados bajo su grado de importancia en una escala Likert de 5 ítems (5. indispensable, 4. importante, 3. neutral, 2. algo importante y 1. no importante) al desarrollar un emprendimiento con ayuda de herramientas tecnológicas (ver figura 2).

\section{Figura 2}

\section{Evaluación de elementos propuestos para emprender con herramientas tecnológicas}

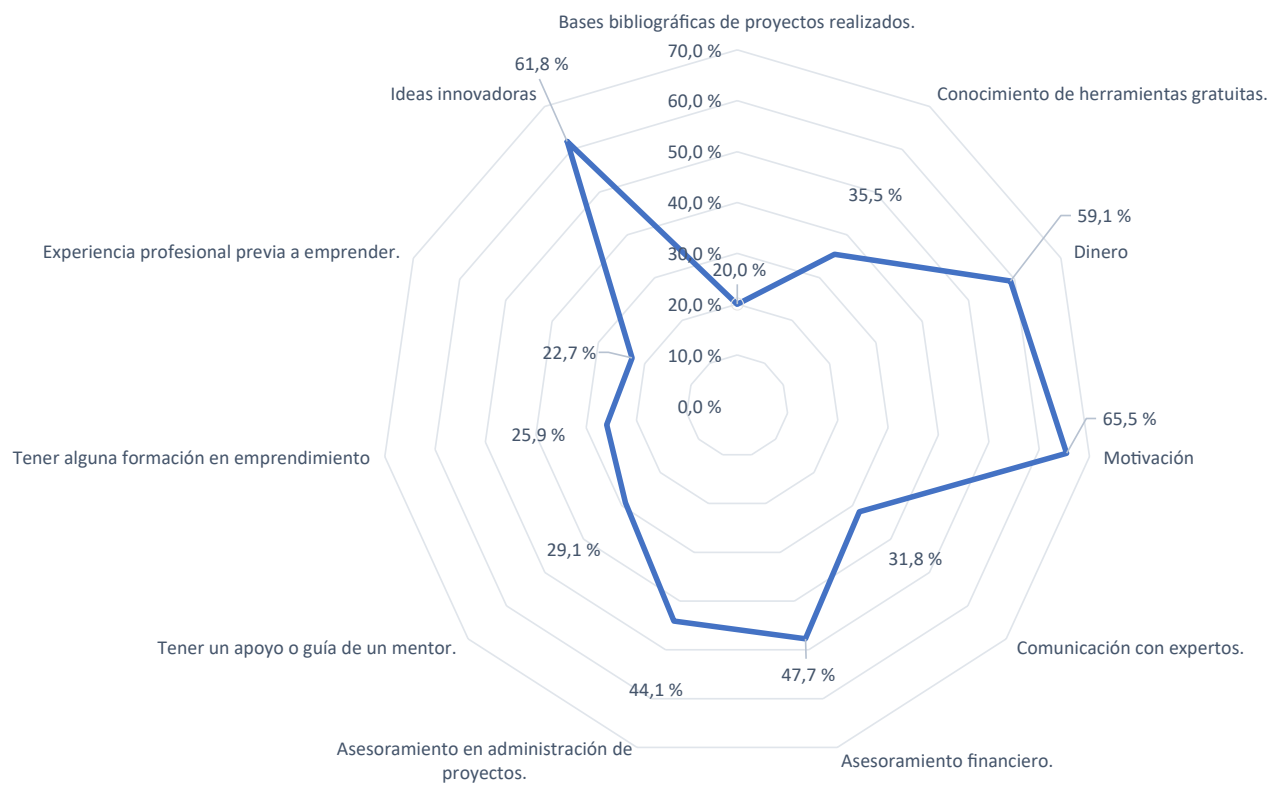

Fuente: elaboración propia.

En la figura 2, se muestra que el elemento con la calificación más alta fue el de la motivación, con un $65,5 \%$ en promedio; en el segundo punto de mayor importancia se encuentran las ideas innovadoras, con un 61,8 \%, y en un tercer grado el dinero, por lo cual se podría indicar que, en un mercado tan digitalizado como el de hoy y tan afectado por la pandemia de la Covid-19, primero se requiere motivación e interés, para luego contextualizar una idea de negocio y finalmente buscar un financiamiento, sea interno o externo.

Ahora bien, los sujetos investigados reiteran el asesoramiento financiero como elemento clave, con un 47,7 \%; el asesoramiento en la administración de proyectos, con un 44,1 \%; y con un 35,5\%, el conocimiento de herramientas digitales gratuitas. Por último, se señala que los elementos para emprender que recibieron la menor calificación fueron la comunicación con expertos, 31,8 \%; tener un apoyo o guía de un mentor, 29,1 \%; contar con alguna formación en emprendimiento, 25,9\%; experiencia profesional previa a emprender, $22,7 \%$; y base de datos, con un $20 \%$. 
Por otra parte, según los datos de la figura 3, las personas encuestadas manifestaron que, para que un emprendedor pueda tener éxito en tiempos de la COVID-19, debe considerar el manejo de conocimientos en marketing y marketing digital; el 67,3\% (148 en términos absolutos) indicó esta condición. El 49, 5\% señaló la necesidad de una estrategia empresarial o plan de negocios y un 31,8 \% técnicas o habilidades de ventas. Por otro lado, con porcentajes menores al $30 \%$, quedaron variables como conocimientos en redes sociales, contabilidad o finanzas, e informática o sistemas.

\section{Figura 3}

Percepciones de conocimientos previos para emprender en tiempos de Covid-19

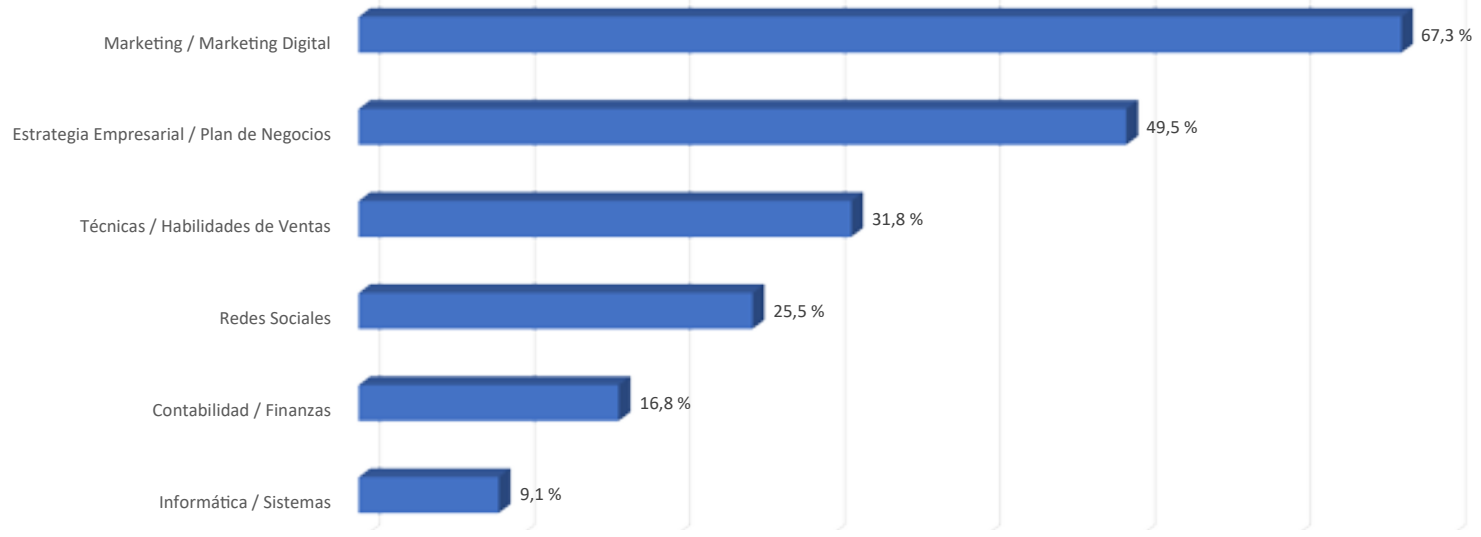

Fuente: elaboración propia.

Paralelamente, a los sujetos investigados se les consultó con respecto a los desafíos más grandes que podrían enfrentar los emprendedores en tiempos pandémicos, a lo cual el 60 \% (132 en número absolutos) se inclinó por la falta de presupuesto, donde resulta cierto indicar que el motivo principal ha sido por todas las medidas de restricción impuestas por las autoridades del Ministerio de Salud de Costa Rica. Así mismo, otros desafíos que se mencionan son los pocos clientes, con un 47,3\%; no contar con el conocimiento y la experiencia, y la falta de confianza y motivación, ambas con un 27,3\%; procesos legales y constitución del negocio, con 26,4 \%; los impuestos, 23,6 \%; colaboradores enfermos y falta de personal capacitado en TIC, ambas con un 13,2 \%; y, por último, la falta de ideas, 15,5\%.

Al retomar el desafío de la falta de personal capacitado en TIC, se consultó acerca de por qué las personas encuestadas no se han capacitado en herramientas digitales, en particular ahora, cuando resulta claro que la Covid-19 aceleró la economía digital. Los resultados son interesantes: alrededor del 30,9 \% desconoce las instituciones que imparten capacitaciones, un $22,7 \%$ no ha encontrado el curso adecuado, el 17,7 \% no se ha capacitado por falta de tiempo, el 8,6 $\%$ lo considera muy caro y $20 \%$ da otras razones como, por ejemplo, que no hay cursos a los cuales se pueda optar, no tienen interés en capacitarse, no lo necesitan o bien que es pérdida de tiempo. En este contexto, se puede resumir que hay ausencia de una verdadera transformación digital en tiempos de la COVID-19.

Sin embargo, en relación con la idea anterior, los sujetos investigados comprenden lo indispensable que es emprender con herramientas tecnológicas durante la pandemia. Cerca del 96,4 \% manifestó su conocimiento en el uso de las redes sociales (Facebook, Instagram, Twitter, WhatsApp), páginas web y correo electrónico. Sin embargo, apenas el $3,6 \%$ conoce de otras aplicaciones gratuitas como, por ejemplo, WA Business o LinkedIn. 
En función de lo planteado, un 70,5 \% de los sujetos en estudio manifestaron que, en tiempos de la COVID-19, la mejor opción para mantener un negocio o, en su defecto, llegar a nuevos clientes, es por medio de las redes sociales en toda su extensión, sin dejar de lado la aplicación de WhatsApp, con un 23,6 \%; otras herramientas de publicidad, con un 4,1\%; y, finalmente, el correo electrónico, con 1,8\%.

Dentro de este orden de ideas, en la figura 4 se estableció un análisis en el cual se determinó cuáles son las habilidades blandas indispensables para emprender un negocio con apoyo de las tecnologías de información y comunicación.

\section{Figura 4}

Habilidades blandas propuestas para emprender con TIC en tiempos de la COVID-19

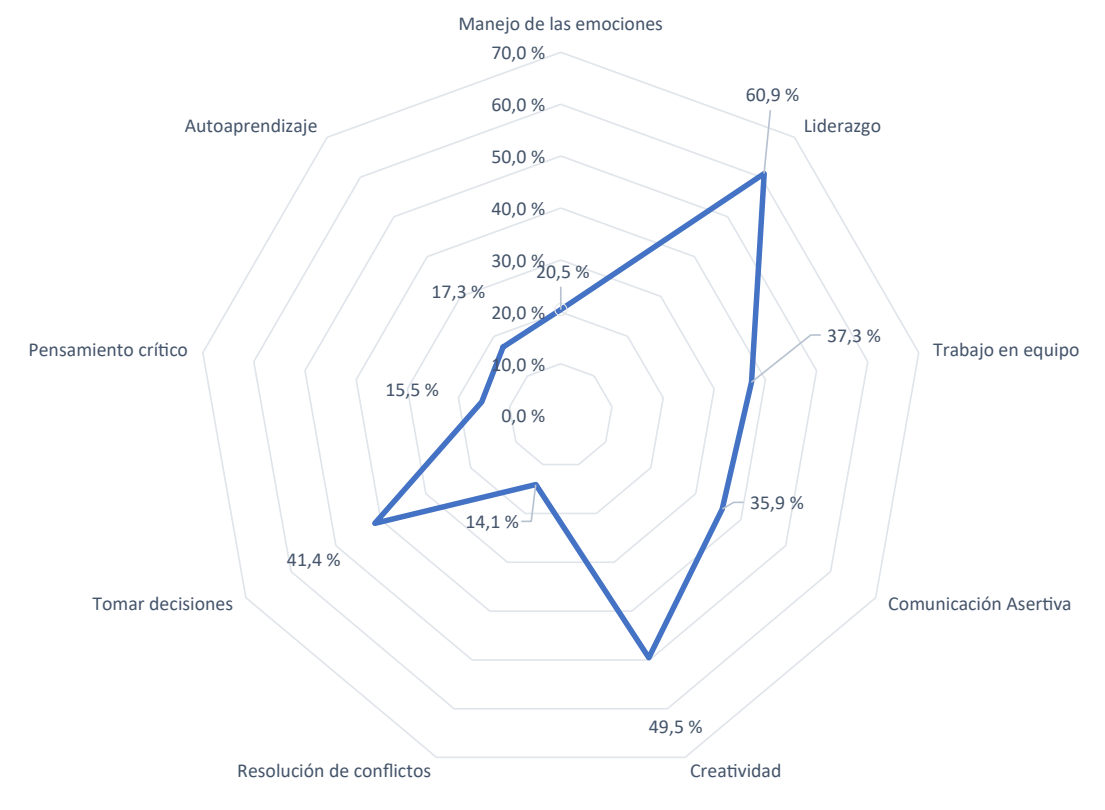

Fuente: elaboración propia.

La figura 4 señala que la habilidad blanda con mayor calificación fue el liderazgo, con un 60,9 \%; sin duda, se pone en evidencia su práctica para todo emprendedor en tiempos de coronavirus. Luego de este, se destaca la importancia de la creatividad, 49,5 \%; la capacidad de tomar decisiones, 41,4\%; las habilidades de trabajo en equipo, $37,3 \%$; y comunicación asertiva, 35,9 \%. Las habilidades blandas con menor calificación, pero no menos importantes, fueron el manejo de las emociones, 20,5\%; el autoaprendizaje, 17,3\%; el pensamiento crítico, 15,5\%; y, finalmente, la resolución de conflictos, $14,1 \%$. 
La figura 5 ilustra las habilidades técnicas o duras, indispensables para emprender con herramientas tecnológicas en tiempos de la COVID-19. Se destaca que el manejo del marketing es el conocimiento por excelencia de la población en estudio, con un 74,5\%, además del manejo de herramientas digitales, con un 73,6\%; todo esto con respecto a la adaptabilidad a un mercado que demanda las capacidades tecnológicas. También destaca el bilingüismo (inglés), con $54,1 \%$, sobre todo en una zona donde la mayoría de los ingresos son captados del turismo, como es la provincia de Guanacaste. Finalmente, se muestra la necesidad del manejo de paquetes de ofimática, con 46,4 \%; el buen manejo y control de las ventas, $15 \%$; análisis estadístico, $12,7 \%$; licencia de conducir, $11,4 \%$; y con 3,6 \%, otros.

\section{Figura 5}

Habilidades duras propuestas para emprender en tiempos de la COVID-19 con TIC

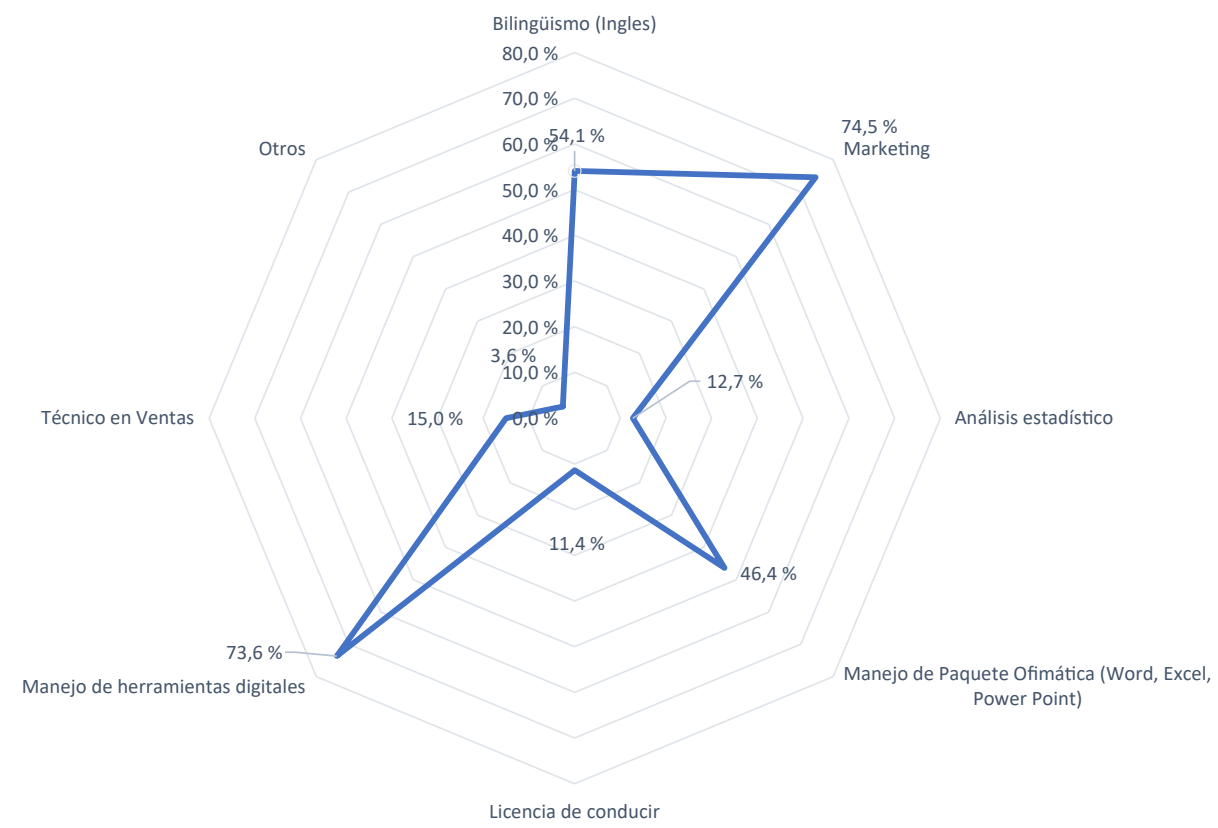

Fuente: elaboración propia.

Por otra parte, en un acercamiento más cualitativo, las personas sujetas al estudio brindaron sus opiniones con respecto a recomendaciones para emprender en tiempos de la COVID-19 con ayuda de las herramientas tecnológicas. A continuación, se recuperan algunas respuestas textuales de dicha recopilación:

- Perseverancia y aprovechar oportunidades para mejorar en la tecnología con las nuevas tendencias de los clientes.

- Investigación con perseverancia sobre estrategias de negocios.

- Tener una buena página web o redes sociales, con buena información de productos y una publicidad que llame la atención de los clientes; así mismo, una buena calidad del producto a ofrecer.

- Motivación de aprendizaje con herramientas que son las del futuro.

- Trasmitir al cliente la confianza en los productos.

- Desarrollar habilidades de manejo de redes sociales y marketing para atraer a los clientes; generar respaldo y profesionalismo en la publicidad de sus ventas para adquirir confianza de los compradores.

- Apoyarse en el uso de las redes sociales y compras en línea; así, los clientes no tendrán que ir a los locales; ofrecer envíos gratis de sus compras. 
- Tener conocimiento en el manejo de las redes sociales, herramienta con la cual las personas dan a conocer sus productos; realizar rifas por medio de estas redes, para darse a conocer.

- Apoyarse en la tecnología y redes sociales, mantener el contacto con los clientes por estos medios y actualizar constantemente la información.

- Realizar estrategias innovadoras por medio de redes sociales para generar al cliente un valor agregado.

- Realizar una buena publicidad de la mano de las redes sociales, mediante los dispositivos móviles.

- Atención rápida por redes sociales y WhatsApp.

- Autocapacitarse con tutoriales para utilizar las herramientas tecnológicas, redes sociales y marketing digital.

- Capacitarse más y aprovechar al máximo la tecnología.

- Capacitarse en el uso del marketing digital y buscar asesoría al iniciar un emprendimiento.

- Manejar el marketing digital y las diferentes formas de publicidad.

- Invertir en la imagen del emprendimiento con fotos, un logo adecuado, material digital para redes, creación de página web, buen servicio al cliente, base de datos para agilizar compras y realizar promociones.

- Tener una mejor administración de las plataformas digitales.

- Capacitarse para desarrollarse de la mejor manera en el área que desea emprender.

- Motivarse y capacitarse en comercio electrónico.

- Buscar ayuda y asesoramiento o capacitarse sobre distintos temas.

- Hacer un estudio del campo y verificar cuáles productos se están vendiendo.

- Saber entender las necesidades del mercado actual, tiendas virtuales e interacción con los clientes.

- Rapidez en la atención al cliente y ofertas.

- Ser asertivo con el logotipo, para trasmitir la verdadera esencia de la marca.

- Utilizar cuentas seguras para el manejo de la información, tanto del cliente como de la propia.

- Trasmitir la confianza en los productos a los clientes.

- Mantenerse actualizado, estar activo en las redes y buscar la automotivación.

- Tener claro el producto o servicio y el mercado al cual desea integrarse.

- Tener una buena técnica de servicio al cliente, en la cual se pueda enfocar en las ayudas tecnológicas, como servicio de ayuda por WhatsApp, soporte en Facebook e información por correo electrónico, entre otros.

- Ser innovador al utilizar las TIC en su beneficio.

- Crear un plan estratégico y contar con asesoría financiera.

- Edición de videos llamativos para la venta de los artículos y, al mismo tiempo, realizar publicidad para la empresa.

- Detectar posibles necesidades y satisfacerlas de manera original y creativa, además de estar en constante capacitación y ser muy dinámico.

- Páginas web que permitan ofrecer y vender el producto en línea.

- Que la idea o producto que vaya a ofrecer sea dirigido al público en general, no frenar la demanda hacia un nicho de mercado en específico, mucha publicidad.

- Ser constantes; tener un buen uso de herramientas tecnológicas es el principal objetivo para mantener un buen negocio en estos tiempos.

- Conseguir muchos clientes en las redes, y publicar precios, estrategias, cómo trabaja la empresa y otros detalles. Además, promocionar con sorteos y premiar a los clientes.

- Más accesibilidad en la creación y uso de las aplicaciones utilizadas para las ventas; tutoriales para personas no adaptadas al cambio virtual. 
- El valor o recurso humano de uno es esencial siempre, en todo ámbito de la vida.

- Perder el miedo al fracaso, estar bien informado del servicio o producto que va a brindar, conocer su cliente meta.

- Capacitarse y buscar el mercado que quiere para el emprendimiento.

- Ser más creativos y valientes, aprovechar esta situación y emprender en las cosas que uno desee. Vivimos en una era digital y, como parte de ella, debemos sacarle el máximo provecho.

- Adaptarse al entorno para ver si es rentable y así emprender, innovar y tener flexibilidad a los cambios.

- Hacer un plan de acción y definir a cuál mercado se quiere ingresar o abarcar.

- Las TIC son un elemento a utilizar para el éxito del negocio; por lo tanto, se requiere capacitación y conocimiento de herramientas gratuitas.

- Desarrollar la habilidad de trabajar con elementos tecnológicos y aprender sobre marketing.

- Que sea algo innovador, ya que en estos tiempos cuesta llamar la atención de los clientes.

- Una recomendación más para la parte legal: en muchas de las ocasiones se imponen trabas para poner un negocio, cuando deberían ser más abiertos para que las personas sientan ese apoyo legal y se motiven, pues lo que necesitan las personas es confianza.

Como resultado de estas percepciones de la población en estudio, en la figura 6 se desprenden las principales similitudes:

\section{Figura 6}

\section{Resumen de las percepciones por la población en estudio}

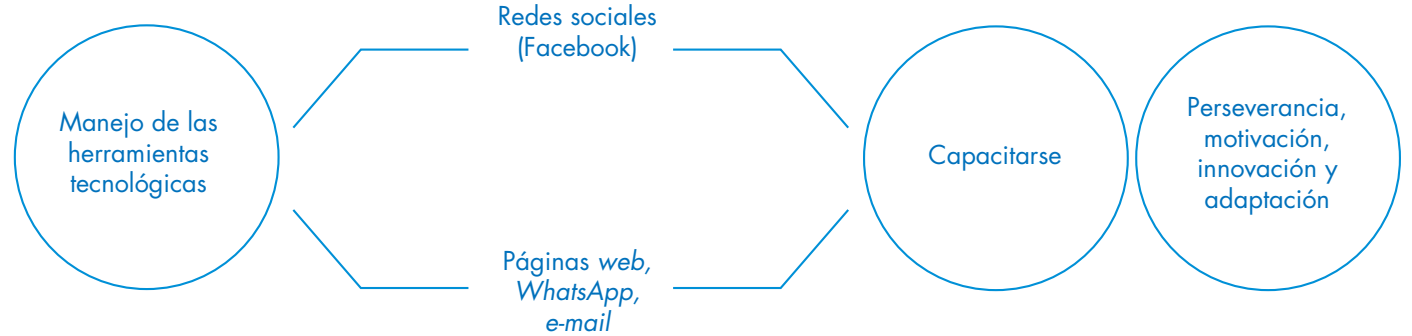

Fuente: elaboración propia.

Se concluye que todas estas variables se adaptan a la situación pandémica actual. De ahí que la demanda y oferta de productos y servicios se muevan con dependencia en un comercio cada vez más electrónico, donde tres de los factores protagonistas son los siguientes: a) el buen uso de las herramientas tecnológicas; b) La virtud de capacitarse y c) las variables de perseverancia, motivación y adaptabilidad a un entorno cambiante, las cuales podrían acercarnos a lograr el éxito del sueño emprendedor en tiempos de la COVID-19. Con respecto al buen uso de las herramientas tecnológicas, coincidimos con Müller (2017), quien afirma que: "a las aplicaciones hay que verlas en contexto. Creer que ellas solas nos llevarán al éxito es tensionar el modelo de negocio y nuestra estrategia” (p. 34). 


\section{CONCLUSIONES}

Los resultados muestran que la utilización de las TIC como herramienta de apoyo sin duda impulsan el emprendimiento y la sostenibilidad de los negocios. Asimismo, la población encuestada percibe que uno de los elementos clave para propiciar la capacidad de emprender es asesorarse con respecto a marca, planificación y costos, además de manejar conocimientos en marketing.

Desde esta perspectiva general y en relación con el impacto de las herramientas tecnológicas en el emprendimiento, se debe considerar que los emprendedores abordan sus decisiones de establecer empresas no de forma solitaria, sino que toman en cuenta elementos derivados del contexto tecnológico y factores que les permitan insertarse de forma competitiva en el mercado global. Por ende, las capacidades para fomentar el ánimo del emprendedor deberán incluir un balance de elementos como las leyes sobre las TIC y los estímulos para la utilización de estas.

Por otra parte, se pone en evidencia que la motivación es un punto alto por considerar para emprender, junto a la necesidad de contar con ideas innovadoras impulsadas desde las herramientas digitales que han provocado un cambio exponencial del giro de los negocios a causa del confinamiento por la COVID-19. De allí que los relatos de encuestados comprueban cómo los seres humanos poseen múltiples impulsos, miedos, estrés y formas de resolverlos.

Dentro de este orden de ideas, se analizaron las percepciones del emprendimiento con apoyo de herramientas tecnológicas en medio de la crisis sanitaria en Costa Rica. Se conoce que un gran porcentaje de los entrevistados hace frente a la pandemia tomando en cuenta sus limitaciones y los escasos recursos que tienen disponibles. A pesar de sus dificultades económicas, se denota que muchos afrontan la pandemia desde una visión positiva, es decir, transforman la situación de crisis en una oportunidad y dan origen a la puesta en práctica a sus iniciativas como proyectos concretos en condiciones adversas.

Ahora bien, esta investigación muestra que el desafío más grande que enfrentan los emprendedores en tiempos pandémicos es la falta de presupuesto, principalmente por causa de las duras medidas de restricción sanitarias impuestas por las autoridades de salud, las cuales buscan mitigar mayores contagios en la población costarricense.

Se concluye que, según el análisis de resultados de esta investigación, existen competencias que fueron reiteradas por los sujetos investigados y que propician adaptarse a la situación pandémica actual: la motivación, el liderazgo, la creatividad, el uso del marketing digital, el manejo de redes sociales y herramientas tecnológicas (Facebook, WhatApps, Instagram, Wa Business, páginas web y correo electrónico, entre otras). Así mismo, otros elementos mencionados, como lo son principalmente asesorías con respecto a marca, planeación y costos, asesoramiento financiero y propuestas de valor innovadoras, entre otros.

Finalmente, resulta crucial, para emprender en el contexto de la COVID-19, potencializar el surgimiento de nuevas ideas, donde se combinen adecuadamente los ingredientes tecnológicos con el acompañamiento y la experiencia de algún mentor. Al mismo tiempo, se debe proceder ágilmente para que los emprendedores logren constituir parte de la solución como una salida de la crisis. Salvaguardar a las nuevas empresas y resguardar su organización y capital humano, así como su posición innovadora, permitirá acelerar la restauración social y económica. 


\section{REFERENCIAS}

Alean P. A., Del Rio, J., Simancas T., R., y Rodríguez A. C. (2017). ¿El emprendimiento como estrategia para el desarrollo humano y social? Saber, Ciencia y Libertad, 12(1), 107-123. https://doi.org/10.18041/2382-3240/ saber.2017v12n1.1470

Álvarez-Vásquez, H. S., y Villafana-Del-Rosario, N. L. (2020). La caja del emprendedor: estrategia periodística sobre emprendimiento y finanzas personales para mercado negro (Tesis de licenciatura). https://repositorio.ulima.edu.pe/ bitstream/handle/20.500.12724/12064/Alvarez_\%20Vasquez_Villafana_Del_Rosario.pdf?sequence=1

Angelelli, P., Hennessey, M., Henríquez, P., Benavente, J. M., Radaelli, V., Sasso, S., Anta, R., Crespi, G., Navarro, J. C., y Vargas, F. (2020)._Respuestas al COVID-19 desde la ciencia, la innovación y el desarrollo productivo_(G. Rivas y C. Suaznabar, eds.). Inter-American Development Bank. https://doi.org/10.18235/0002347_

Barrantes Echeverría, R. (2015). Métodos de estudio a distancia e investigación: a la búsqueda del conocimiento científico. EUNED.

Barrera Agudelo, V. F., Luna Losada, S. M., y Molano Buenaventura, J. P. (2020). Oportunidades de emprendimiento en Bogotá originadas por cambios de hábitos de consumo debido a la pandemia. Universidad Ean. http://hdl.handle. net/10882/10185

Chaves N. y Fonseca H. (2015). Emprendedurismo en Costa Rica: estancamiento en la transición a la innovación. Economía y Sociedad, 20(48), 1-19. https://doi.org/10.15359/eys.20-48.5

Félix, A. G., y García, N. G. (2020). Estudio de pérdidas y estrategias de reactivación para el sector turístico por crisis sanitaria Covid-19 en el destino Manta (Ecuador). Revista Internacional de Turismo, Empresa y Territorio. RITUREM, 4(1), 79-103. https://doi.org/10.21071/riturem.v4i1.12743

Gonzaga Añazco, S. J., Alaña Castillo, T. P., y González Ordóñez, A. I. (2017). Competitividad y emprendimiento: herramientas de crecimiento económico de un país. INNOVA Research Journal, 2(8.1), 322-328. https://doi. org/10.33890/innova.v2.n8.1.2017.386

González, E., Olivié, C., Maio, A., Quiñones, Y., Jiménez, T., Lucía, L., y García-Arranz, A. (2018). Empresa familiar, emprendimiento e intraemprendimiento. EAE Business School. https://www.laempresafamiliar.com/wpcontent/uploads/2019/01/SRC_Emprendimiento_EmpresaFamiliar-1.pdf

Hernández Sampieri, R.; Fernández, C. y Baptista, M. (2014). Metodología de la Investigación McGraw-Hill Education.

Hoyos-Estrada, S., y Sastoque-Gómez, J. (2020). Marketing Digital como oportunidad de digitalización de las PYMES en Colombia en tiempo del Covid -19. Revista Científica Anfibios, 3(1), 39-46. https://doi.org/10.37979/ afb.2020v3n 1.60

Jaramillo, M., y Ñopo, H. (2020). Covid-19 y el shock externo: Impactos económicos y opciones de política en el Perú | PNUD en América Latina y el Caribe. UNDP. https://wwwlatinamerica.undp.org/content/rblac/es/home/ library/crisis_prevention_and_recovery/covid-19-y-el-schock-externo--impactos-economicos-y-opciones-de-. html?fbclid=IwAR2MpysdoS_1hd-Z_5lrvC983Bd4ItLj_rc729cjS2QADZyl2Sn3S-btjKM

Linares, R. R. (2020). La Pyme post COVID 19: ¿seguirá siendo un comodín entre el Estado y el Mercado? Catalejo. https://www.academia.edu/download/64347458/La\%20Pyme\%20post\%20COVID\%2019\%20VF\%20120820.pdf

López, G., y Rivera, J. (2020). De la teoría a la práctica: el emprendimiento en Ecuador en época de COVID 19. Investigación y Desarrollo, 12(1), 26-37. https://revistas.uta.edu.ec/erevista/index.php/dide/article/view/982_

Mejías, V. V., Arce, A. V., Sánchez, F. C., y Alfaro, D. Á. (2020). Impacto del COVID-19 en las pymes costarricenses: Un enfoque para sobrellevar la crisis actual. Revista Ventana, 14(1), 39-42. https://revistas.tec.ac.cr/index.php/ ventana/article/download/5456/5187

Müller, C. (2017). Las apps no son sinónimo de emprendimiento: ¡cuidado! IEEM Revista de Negocios, 20(5), 30-34. https://www.hacerempresa.uy/wp-content/uploads/2018/12/IEEM-octubre-Art-apps.pdf 
Nicolás Martínez, C., y Rubio Bañón, A. (2020). Entrepreneurship in times of crisis: An exploratory analysis of the COVID-19's effects. Small Business International Review, 4(2), 53-66. https://doi.org/10.26784/sbir.v4i2.279

Rodríguez, M. D. (2016). Emprendimiento sostenible, significado y dimensiones. Revista Katharsis, 21, 449-479. http:// revistas.iue.edu.co/revistasiue/index.php/katharsis/article/view/775/1066

Romero, M. A., y Milone, M. (2016). El Emprendimiento en España: Intención Emprendedora, Motivaciones y Obstáculos. Journal of Globalization, Competitiveness $\&$ Governability / Revista de Globalización, Competitividad y Gobernabilidad / Revista de Globalização, Competitividade e Governabilidade, 10(1), 95-109. https://www.redalyc. org/pdf/5118/511854473005.pdf

Vásquez-Lafebre, L. M., Quevedo-Barros, M. R., Neira-Neira, M. L., y Quevedo-Vázquez, J. O. (2020). Rol de los emprendimientos y su responsabilidad post crisis económica covid-19. Revista Científica FIPCAEC (Fomento de la investigación y publicación en Ciencias Administrativas, Económicas y Contables). Polo de Capacitación, Investigación y Publicación (POCAIP), 5(3), 723-734. https://doi.org/10.23857/fipcaec.v5i3.257

Vélez, R. X. y Ortiz, R. S. (2016). Emprendimiento e innovación: Una aproximación teórica. Dominio de las Ciencias, 2(4), 346-369. https://dialnet.unirioja.es/descarga/articulo/5802889.pdf

Zamora-Boza, C. (2017). La importancia del emprendimiento en la economía: el caso de Ecuador. Revista Espacios Económicos, 39(8), 15-27. https://www.revistaespacios.com/a18v39n07/a18v39no7p15.pdf 